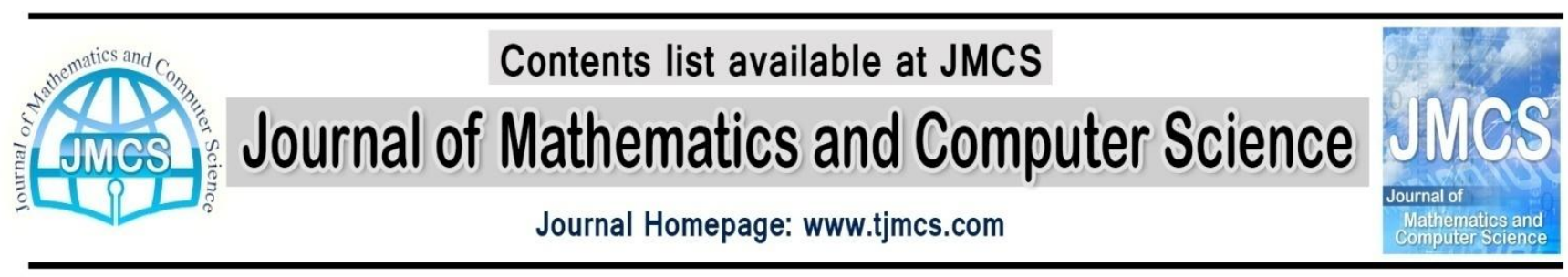

\title{
A Method for Reducing Repetitive items on Weighted Data using the WIT- WFI Algorithm
}

\author{
Akbar Rashidi $^{1}$, Arash Ghorbannia Delavar ${ }^{2}$ \\ Department of Computer, Payame Noor University, \\ PO BOX 19395-3697, Tehran, IRAN \\ rashidi.igs@gmail.com ${ }^{1}$, a_ghorbannia@pnu.ac.ir²
}

Article history:

Received August 2013

Accepted September 2013

Available online September 2013

\begin{abstract}
Trying and mining frequent item sets plays an important role in the mining of association rules in a dataset that stored with items and transactions an items can used for various significance association rules is a important and considerable ways in data mining without presidency . one of discussion that today investigate is mining and finding frequent weighted item set and reduce run time of algorithm and reduce production frequent item sets is one of problem for research at this paper we purpose present some method and ways for reduce run time of algorithm and reduce production frequent item set all methods and ways applying on WIT algorithm and WIT-Tree structure in first section we express and description classic association rule method (Apriori) and then WIT and then WIT-Diff algorithm and finally explain my proposed ways and experimental results.
\end{abstract}

Keywords: Data mining - Frequent items - Weighted item sets - WIT- Tree - Association rules.

\section{Introduction}

Association Rules Mining (ARM) is an important part in the domain of knowledge discovery in data (KDD) [1,2]. Association rule mining is used for finding and mining frequent patterns and relationship between transactions in a database or dataset. Association rules used of presidency learning principle, that purpose the this principle is obvious and we understand that research what knowledge unlike without presidency way than result and purpose the mining not clear. Given a set of items $I=\{i 1, i 2, \ldots$, in $\}$, a transaction is defined as a subset of I. The input to an ARM algorithm is a dataset D comprising a set of transactions. Given an item set $\mathrm{X} \subseteq \mathrm{I}$, the support of $\mathrm{X}$ in $\mathrm{D}$, denoted as $\sigma(\mathrm{X})$, is the number of transactions in D which contain X [18]. An item set is described as being frequent if its support is larger 
than or equal to a user supplied minimum support threshold (min Sup). A "classical" Association Rule $(A R)$ is an expression of the form $\{X \rightarrow Y$ (sup, conf) $\}$, where $X, Y \subseteq I$ and $X \cap Y=\varnothing$. The support of this rule is $\sup =\sigma(X Y)$ and the confidence is $\operatorname{conf}=\frac{\sigma(X Y)}{\sigma(X)}$. Given a specific min Sup and a minimum confidence threshold (min Conf), we want to mine all association rules whose support and confidence exceeds min Sup and min Conf respectively $[3,6]$.

However, Classical association rule have some problem that very great run time and many scan of database for finding item sets if we add computation time of items with weight as time of algorithm raising .purpose the this paper is expansion WIT algorithm for mining frequent items in view run time and reduce product item set. The rest of this paper is organized as follows. Section 2 presents some related work about the mining of frequent weighted items and weighted association rules and some terms and equations. Section 3 we explain WIT-Tree structure and in Section 4 explain WIT algorithm in Section 5 explain and descript WIT-DIFF algorithm .in Section 6 explain and descript my proposal methods. Some experimental results are present in Section 7 .and my conclusion in Section 8.

\section{Related works}

This section presents some related works. The section begins with a formal definition of weighted transaction databases. A weighted transaction database (D) is defined as follows: D comprises a set of transactions $\mathrm{T}=\left\{\mathrm{t}_{1}, \mathrm{t}_{2},, \mathrm{t}_{\mathrm{n}}\right\}$, a set of items $\mathrm{I}=\left\{\mathrm{i}_{1}, \mathrm{i}_{2},, \mathrm{i}_{\mathrm{n}}\right\}$ and a set of positive weights $\mathrm{W}=\left\{\mathrm{w}_{1}, \mathrm{w}_{2},, \mathrm{w}_{\mathrm{n}}\right\}$ corresponding to each item in I. For example, consider the data presented in Tables 1 and 2. Table 1 presents a data set comprising six transactions $T=\left\{t_{1}, t_{2} \ldots t_{6}\right\}$ and five items $I=\{A, B, C, D, E\}$. The weights of these items are presented in Table $2, \mathrm{~W}=\{0.6,0.1,0.3,0.9,0.2\}[4,5]$.

Table 1:The transaction database

\begin{tabular}{ll}
\hline Transactions & Bought items \\
\hline 1 & $A, B, D, E$ \\
2 & $B, C, E$ \\
3 & $A, B, D, E$ \\
4 & $A, B, C, E$ \\
5 & $A, B, C, D, E$ \\
6 & $B, C, D$ \\
\hline
\end{tabular}

Table 2: Items weight

Items weight.

\begin{tabular}{ll}
\hline Item & Weight \\
\hline A & 0.6 \\
B & 0.1 \\
C & 0.3 \\
D & 0.9 \\
E & 0.2 \\
\hline
\end{tabular}

The equations that we used in this paper consist:

- Calculation weight of transaction $\left(\mathrm{tw}^{1}\right)$.

\footnotetext{
${ }^{1}$ Transaction weight
} 
- Calculation weight of items or weighted support (ws).

For acquire weight of a transaction we must sum of all items weight in transaction and then calculate average of items with divide sum of items in count of items in each transactions. See definition 2.1, we can compute the transaction weight $[7,8]$.

$\mathbf{t}_{\mathbf{k}}:$ Transaction $\mathrm{k}$.

$$
\mathrm{Tw}_{\left(\mathrm{t}_{\mathrm{k}}\right)}=\frac{\sum \mathrm{i}_{\mathrm{j}} \in \mathrm{t}_{\mathrm{k}} \mathrm{W}_{\mathrm{j}}}{\left|\mathrm{t}_{\mathrm{k}}\right|} \quad 2.1
$$

$\mathbf{i}_{\mathbf{j}}: \mathbf{j}$ th items in transaction.

$\left|\mathbf{t}_{\mathbf{k}}\right|$ : Size of transaction $\mathrm{k}$, count of items .

$\mathbf{w}_{\mathbf{j}}$ : Weight of $\mathbf{j}$ th item.

For compute weighted support must compute sum of transaction weight (table 3) and divide to sum of transaction. See definition 2.2, we can compute the transaction weight.

$$
\mathrm{WS}(\mathrm{X})=\frac{\sum_{\mathrm{t}_{\mathrm{k}} \mathrm{et}(\mathrm{x})} \mathrm{tw}\left(\mathrm{t}_{\mathrm{k}}\right)}{\sum_{\mathrm{t}_{\mathrm{k}} \mathrm{ET}} \mathrm{tw}\left(\mathrm{t}_{\mathrm{k}}\right)} \quad 2.2
$$

$\mathrm{X}$ : The item.

$\operatorname{tw}\left(t_{k}\right)$ : Weight of transaction $t_{k}$.

$\mathrm{t}(\mathrm{X})$ : Transaction consist item (X).

T: total of dataset.

Table 3: Transaction weight for transaction in table 1

Transaction weights for transactions in Table 1.

\begin{tabular}{ll}
\hline Transactions & $t w$ \\
\hline 1 & 0.45 \\
2 & 0.2 \\
3 & 0.45 \\
4 & 0.3 \\
5 & 0.42 \\
6 & 0.43 \\
Sum & 2.25 \\
\hline
\end{tabular}

Example for calculation transaction weight and weighted Support. For transaction weight used of table 1 , table 2 and definition 2.1, we can compute the transaction weight:

$$
\mathrm{tw}=\frac{0.6+0.1+0.9+0.2}{4}=0.45
$$


And Table 3 shows all tw values of transactions in Table 1.

From Tables 1 and 3, and Definition 2.2, we can compute the ws(BD) value as follows: Because BD appears in transactions $\{1,3,5,6\}$, ws(BD) is computed:

$$
\mathrm{WS}(\mathrm{BD})=\frac{0.45+0.45+0.42+0.43}{2.25} \approx 0.78
$$

From equation 2.1 and 2.2 using in all algorithm and my proposal methods .in all methods a input value (threshold) used for filter algorithm input, we used this value for comparison and evaluation run time of algorithm and methods. The mining of FWI requires identification all item sets whose weighted support satisfies a user specified minimum weighted support threshold $\left(\right.$ minws $\left.^{2}\right)$.

$$
\mathrm{FWI}=\{\mathrm{X} \subseteq \mathrm{I} \mid \mathrm{WS}(\mathrm{X}) \geq \operatorname{minWS}\} \quad 2.3
$$

\section{WIT-Tree structure}

The structure that we used to explain my methods is WIT structure for description this structure we first explain some terms [9].

$\mathrm{X}$ : set of items.

$t(X)$ : Set of transaction contain item(X).

ws: Value of weighted support for item(X).

for show a node of my tree used $\langle\mathrm{X}, \mathrm{T}(\mathrm{x})$,ws $>$ style. In this style $\mathrm{x}$ is my item and $\mathrm{T}(\mathrm{x})$ is transactions id which item $\mathrm{X}$ member of them. And ws is value of weighted support. For more description see Figure 1.

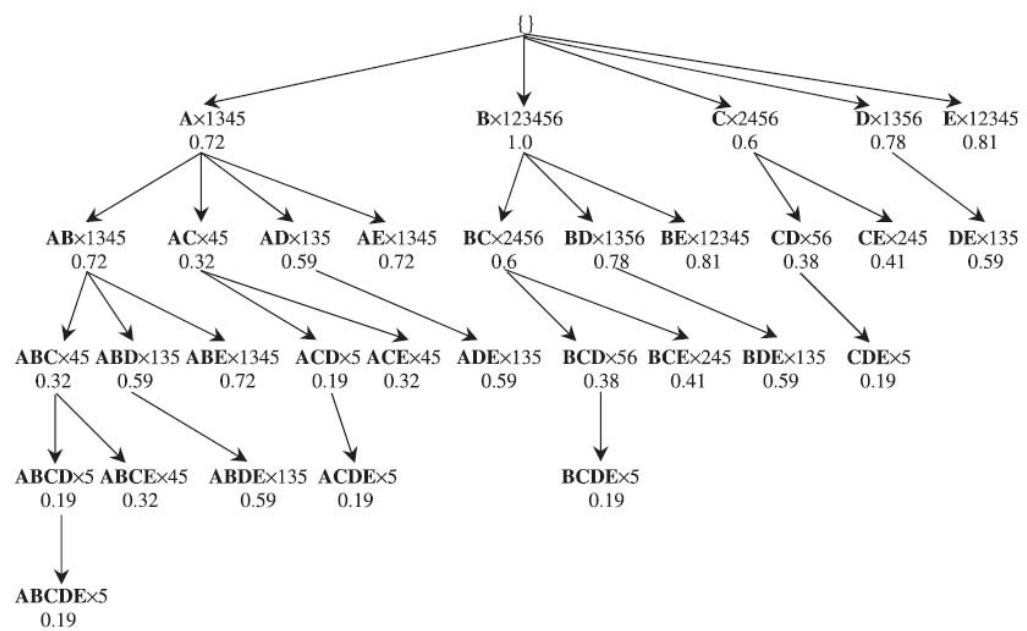

Figure 1: WIT-Tree structure example

In fig 1 level $\varnothing$ or root display with two accolade \{\} .if items member of a transaction consist same prefix they are called equivalence class items and display them with [ ], for example if item $\mathrm{X}$ is prefix item in some items we illustrate it with [X].in root level my prefix item is $\emptyset$ and equivalence class is

\footnotetext{
${ }^{2}$ Minimum weighted support
} 
$[\varnothing]$.in this figure we see for all levels and items we compute transaction weight and weighted support. For compute number of all items in dataset used of $2^{\mathrm{k}}-1$ equation that variable $\mathrm{k}$ is all items in dataset and too for number of items in each level we used of combination equation $\left(\begin{array}{l}k \\ L\end{array}\right)$.variable $k$ is number of items and variable $\mathrm{L}$ is level numeral [2,19].

In this structure for create next level must used of two top level items or parent item. For example level 1 on fig 1 consist $\{\mathrm{A}, \mathrm{B}, \mathrm{C}, \mathrm{D}, \mathrm{E}\}$ items and a part of level 2 consist items with equivalence class [A] ,$\{\mathrm{AB}, \mathrm{AC}, \mathrm{AD}, \mathrm{AE}\}$.this process resume for all items. Inspection of Fig. 1 suggests that all item sets satisfy the downward closure property. So, we can prune an equivalence class in the WIT-tree if its ws value does not satisfy the minws. For example, suppose that minwus $=0.4$, because ws $(\mathrm{ABC})=0.32<$ minws we can prune the equivalence class with the prefix $\mathrm{ABC}$, i.e., all child nodes of $\mathrm{ABC}$ can be pruned. In nest sections explain WIT and WIT-Diff methods.

\section{WIT algorithm}

In classic association rule (Apriori) for mining frequent weighted items we must for all items compute transaction weight and ws and too scan dataset for transaction id and location of each item in dataset. But in WIT we proceed to reduce repetitive calculation and whereupon reducing run time of my algorithm and methods. For this purpose we explain some theorems. we propose algorithms for mining FWI from weighted transaction databases [11,12]. First an algorithm for directly mining FWI from WIT-trees is presented. It uses a minws threshold and the downward closure property to prune nodes that are not frequent. Some theorems are then derived and based on these theorems, an improved algorithm is proposed. Finally, the algorithm is further developed, by adopting a Diffset strategy to allow for fast computating the weighted support of item sets in a memory efficient way. If we have two item set X,Y that transaction id of item set $\mathrm{X}$ equal item set $\mathrm{Y}$, otherwise $\mathrm{t}(\mathrm{X})=\mathrm{t}(\mathrm{y})$.in result can deduction for two item set $\mathrm{X}$ and $\mathrm{Y}$, value of Weighted support is equal. Or otherwise WS $(\mathrm{X})=\mathrm{WS}(\mathrm{Y})$.

$$
\text { If } \mathrm{t}(\mathrm{X})=\mathrm{t}(\mathrm{Y}) \quad \text { Then } \quad \mathrm{WS}(\mathrm{X})=\mathrm{WS}(\mathrm{Y})
$$

If item set $\mathrm{X}$ member of collection $\mathrm{Y}$ and too number of transaction common together equal, in result weighted support value of two item set $\mathrm{X}$ and $\mathrm{Y}$ is unify.

$\begin{aligned} \text { if } \quad \mathrm{X} \subseteq \mathrm{Y} \quad \text { and } \quad|\mathrm{t}(\mathrm{X})|=|\mathrm{t}(\mathrm{Y})| & 4.2 \\ & \text { then } \mathrm{WS}(\mathrm{X})=\mathrm{WS}(\mathrm{Y})\end{aligned}$




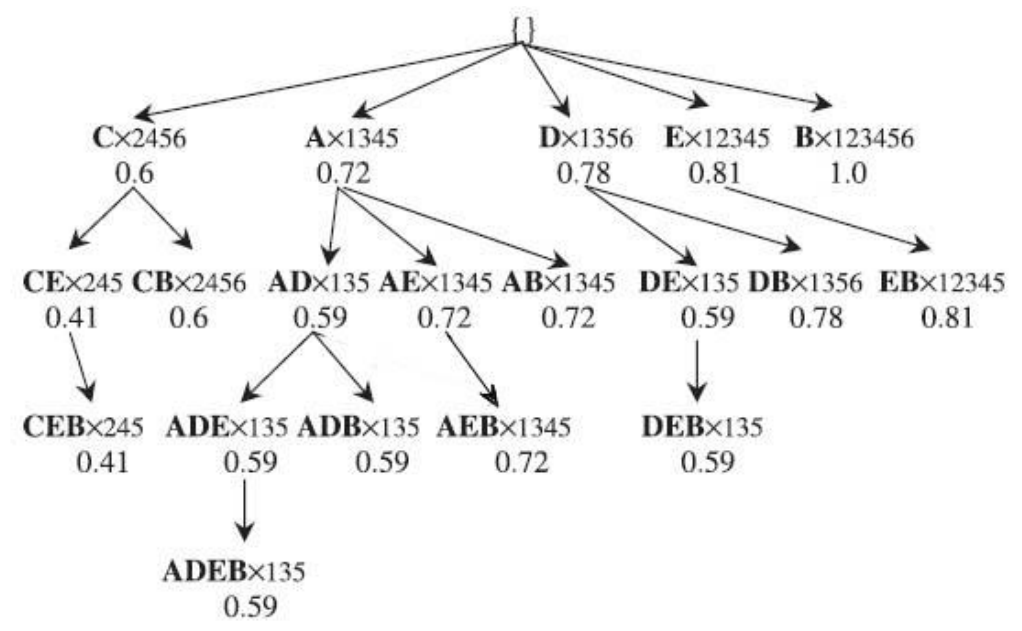

Figure 2: Example of WIT

For more description can see figures 2 and 3.

In Fig 2, first selection all items that satisfy min $\mathrm{ws}^{3}$ and then sort them with increasing by their weighted support and set them in frequent weighted items (FWI) list. In line 4 of WIT algorithm Fig 3 call extend function for produce next item set from combination top level items. Too equation 4.1 and 4.2 used in $9,10,11$ lines. With inspection In fig 2 for item $<A, 1345,0.72>$ and that combination with item set $\langle\mathrm{B}, 123456,1\rangle$ and item set $\angle \mathrm{AB}, 1345$,? $\rangle$ value of weighted support of item set $\mathrm{AB}$ with definition 4.1 and 4.2 equal with item $A$ weighted support and not require for computing ws for item set $\mathrm{AB}$.this process continue for next level and item sets to pending remain one item set in level.

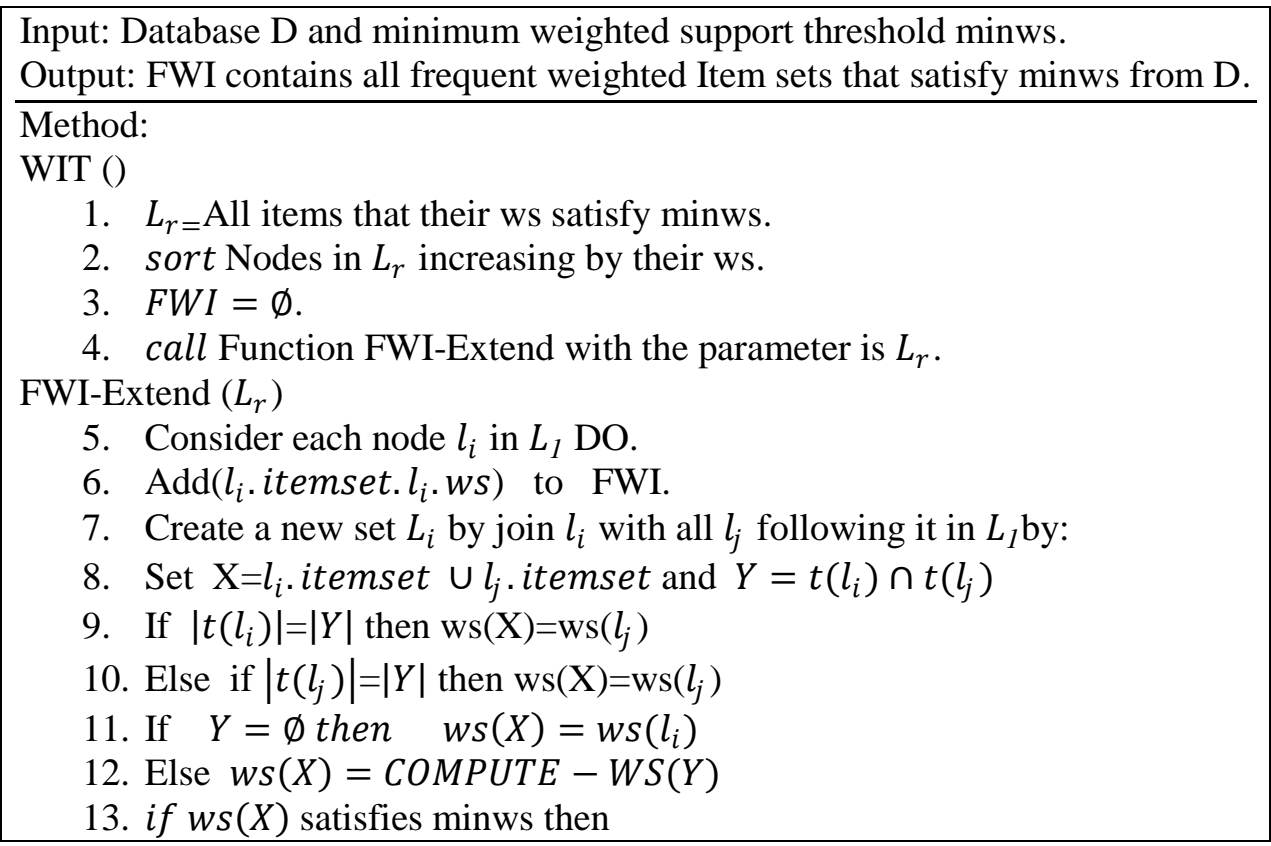

\footnotetext{
${ }^{3}$ Weighted Support
} 
14. Add new Node $<X, Y, w s(X)>$ into $L_{i}$

15. if number of nodes in $L_{i} \geq 2$ then

16. Call recursive the function FWI-Extend with the parameter is $L_{i}$

Figure 3: WIT algorithm

\section{WIT-Diff algorithm}

Proposed the Diffset ${ }^{4}$ strategy for fast computing the support of item sets and saving memory to store Tidsets $^{5}$. We recognize that it can be used for fast computing the ws values of item sets (1). Diffset computes the difference set between two Tidsets in the same equivalence class. In a dense database, the size of Diffset is smaller than the Tidset. Thus, using Diffset will consume less storage and allow for the fast computing of weighted support values. In this algorithm difference between PX and PY illustrated $\mathrm{d}(\mathrm{PXY})$ that $\mathrm{X}$ and $\mathrm{Y}$ are my items and $\mathrm{P}$ is prefix that illustrated equivalence class of items $[13,15]$.(Fig 4)

$$
d(p x y)=\frac{t(p x)}{t(p y)}
$$

If have values of $\mathrm{d}(\mathrm{PX})$ and $\mathrm{d}(\mathrm{PY})$ and I will compute $\mathrm{d}(\mathrm{PXY})$, can used bellow equation:

$$
d(p x y)=\frac{d(p y)}{d(p x)}
$$

Beneficial of equation 5.1 and 5.2 can reach an equation for calculate weighted support in this method:

$$
\mathrm{WS}(\mathrm{pxy})=\mathrm{ws}(\mathrm{px})-\frac{\sum_{\mathrm{t} \in \mathrm{d}(\mathrm{pxy})} \mathrm{tw}(\mathrm{t})}{\sum_{\mathrm{t} \in \mathrm{T}} \mathrm{tw}(\mathrm{t})}
$$

If value of $d(P X Y)=\varnothing$ then value of $w s(P X Y)=w s(P X)$, this denote if $d(P X Y)=\varnothing$ then value of ws is equal parent ws value.

Input: Database D and minimum weighted support threshold minws.

Output: FWI contains all frequent weighted Itemsets that satisfy minws from D.

Method:

WIT-Diff()

1. $L_{r}=$ All items that their ws satisfy minws.

2. sort Nodes in $L_{r}$ increasing by their tid.

3. $F W I=\emptyset$.

4. call Function FWI-Extend-Diff with the parameter is $L_{r}$.

FWI-Extend-Diff $\left(L_{r}\right)$

5. Consider each node $l_{i}$ in $L_{1}$ DO.

6. $\operatorname{Add}\left(l_{i}\right.$. itemset. $\left.l_{i} . w s\right)$ to FWI.

7. Create a new set $L_{i}$ by join $l_{i}$ with all $l_{j}$ following it in $L_{l}$ by:

8. Set $\mathrm{X}=l_{i}$. itemset $\cup l_{j}$. itemset

${ }^{4}$ Difference set

${ }^{5}$ Transaction id number sets 
9. If $L_{r}$ is the first Level Then $Y=\frac{t\left(l_{i}\right)}{t\left(l_{j}\right)}$

10. Else $Y=\frac{d\left(l_{i}\right)}{d\left(l_{j}\right)}$

11. If $Y=\emptyset$ then $\quad w s(X)=w s\left(l_{i}\right)$

12. Else $w s(X)=C O M P U T E-W S-D I F F(Y)$

13. if $w s(X)$ satisfies minws then

14. Add new Node $<X, Y, w s(X)>$ into $L_{i}$

15. if number of nodes in $L_{i} \geq 2$ then

16.Call recursive the function FWI-Extend-Diff with the parameter is $L_{i}$

Figure 4: WIT-Diff algorithm

For description example of this algorithm used of table 1, table 2 and fig 5.for compute difference between item $\mathrm{B}$ and $\mathrm{D}$ we must compute $\mathrm{d}(\mathrm{BD})$ :

$$
d(B D)=\frac{t(B)}{t(D)}
$$

That $t(B)=123456$ and $t(d)=1356$ then difference of two items is 24 , while that result of unlike combination of item $\mathrm{B}$ and item $\mathrm{D}$ is null.

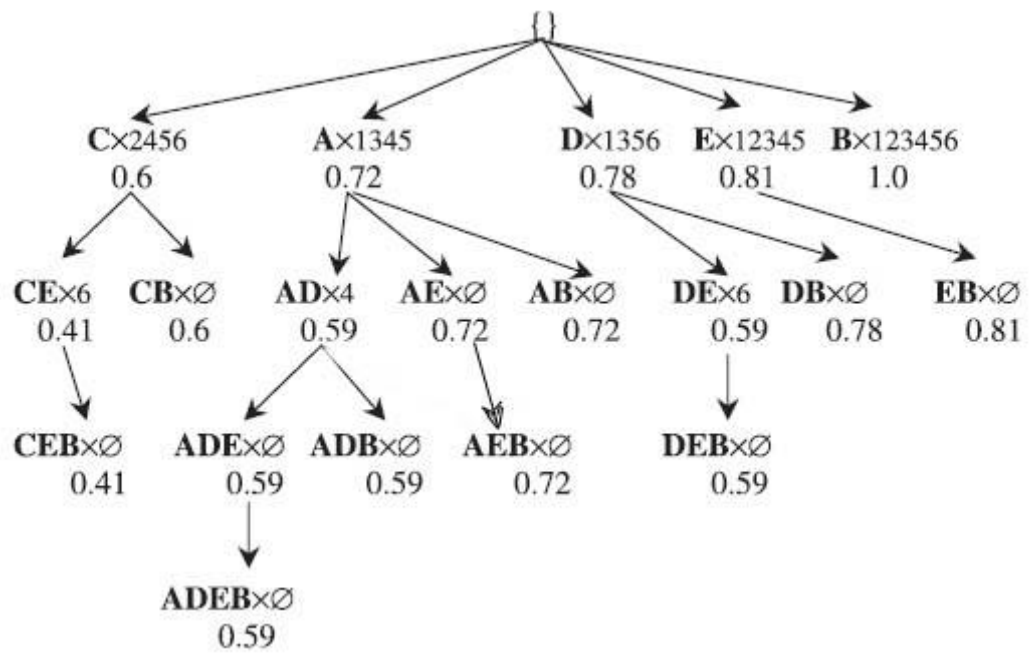

Figure 5: WIT-Diff example

Using the example data presented in Tables 1 and 3, and the algorithm in Fig. 5, we illustrate the WITDIFF algorithm with minws $=0.4$ as follows. Level 1 of the WIT-tree contains single items, their tids ${ }^{6}$, and their ws. They are sorted in increasing order by their |tids|. The purpose of this work is to compute Diffset faster $[16,17]$.

A join D:

$$
d(A D)=\frac{t(A)}{t(D)}=\frac{1345}{1356}=4 \Rightarrow w s(A D)
$$

\footnotetext{
${ }^{6}$ Transaction identity number
} 


$$
=w s(A)-\frac{\sum_{t \in d(A D)} t w(t)}{\sum_{t \in T} t w(t)}=0.72-\frac{0.3}{2.25}=0.59
$$

A join $B$ :

$$
\begin{gathered}
d(A B)=\frac{t(A)}{t(B)}=\frac{1345}{123456}=\varnothing \\
\Rightarrow \quad \text { ws }(A B)=w s(A)=0.72
\end{gathered}
$$

\section{The proposed methods:}

In explained ways and methods we used of datasets that cleaned and removed the duplicate items and preprocessing with other tools such as Microsoft excel ,Clementine, Weka or with other tools. We modified WIT-Diff algorithm at first remove duplicate items in each transactions and reduce transactions weight computations for similar transactions.

\subsection{WIT-Odd or Even method:}

In this way at first scanning dataset and selection all items that not repetitive and unique then classify all items in two group, odd items and even items .in continue of process the algorithm done individually for even items and odd items .and at the end we have two run times, one for even items and other run time for odd items .and have compounds of only odd and even items .in result we reducing run time and produce frequent item sets. (Fig 6)

Input: Database D and minimum weighted support threshold minws.

Output: FWI contains all frequent weighted Itemsets that satisfy minws from D and Even and odd weight.

Method:

WIT-Diff-even and odd()

IF $w_{i}$ MOD $2 \neq 0$ then // odd items.

Else if $w_{i}$ MOD $2=0$ then //even items.

1. $L_{r=}$ All items that their ws satisfy minws.

2. sort Nodes in $L_{r}$ increasing by their ws.

3. $F W I=\emptyset$.

4. call Function FWI-Extend-Diff - even and odd with the parameter is $L_{r}$. FWI-Extend-Diff-even and odd $\left(L_{r}\right)$

5. Consider each node $l_{i}$ in $L_{l}$ DO.

6. Add $\left(l_{i}\right.$. itemset. $\left.l_{i} . w s\right)$ to FWI.

7. Create a new set $L_{i}$ by join $l_{i}$ with all $l_{j}$ following it in $L_{l}$ by:

8. Set $\mathrm{X}=l_{i}$. itemset $\cup l_{j}$. itemset

9. If $L_{r}$ is the first Level Then $Y=\frac{t\left(l_{i}\right)}{t\left(l_{j}\right)}$ 
10. Else $Y=\frac{d\left(l_{i}\right)}{d\left(l_{j}\right)}$

11. If $Y=\emptyset$ then $w s(X)=w s\left(l_{i}\right)$

12. Else $w s(X)=C O M P U T E-W S-D I F F-$ even and odd $(Y)$

13. if $w s(X)$ satisfies minws then

14. Add new Node $<X, Y$,ws $(X)>$ into $L_{i}$

15. if number of nodes in $L_{i} \geq 2$ then

16.Call recursive the function FWI-Extend-Diff -even and odd with the parameter is $L_{i}$

Figure 6: WIT-Diff-even and odd algorithm

\subsection{WIT-Max of Even or odd method:}

The previous method,we use of two variables for keeping count of even and odd items then at the end counting down count of two variables and then each of have maximum count and value ,my algorithm run with it. In this way purpose is reduce more calculations. (Fig 7)

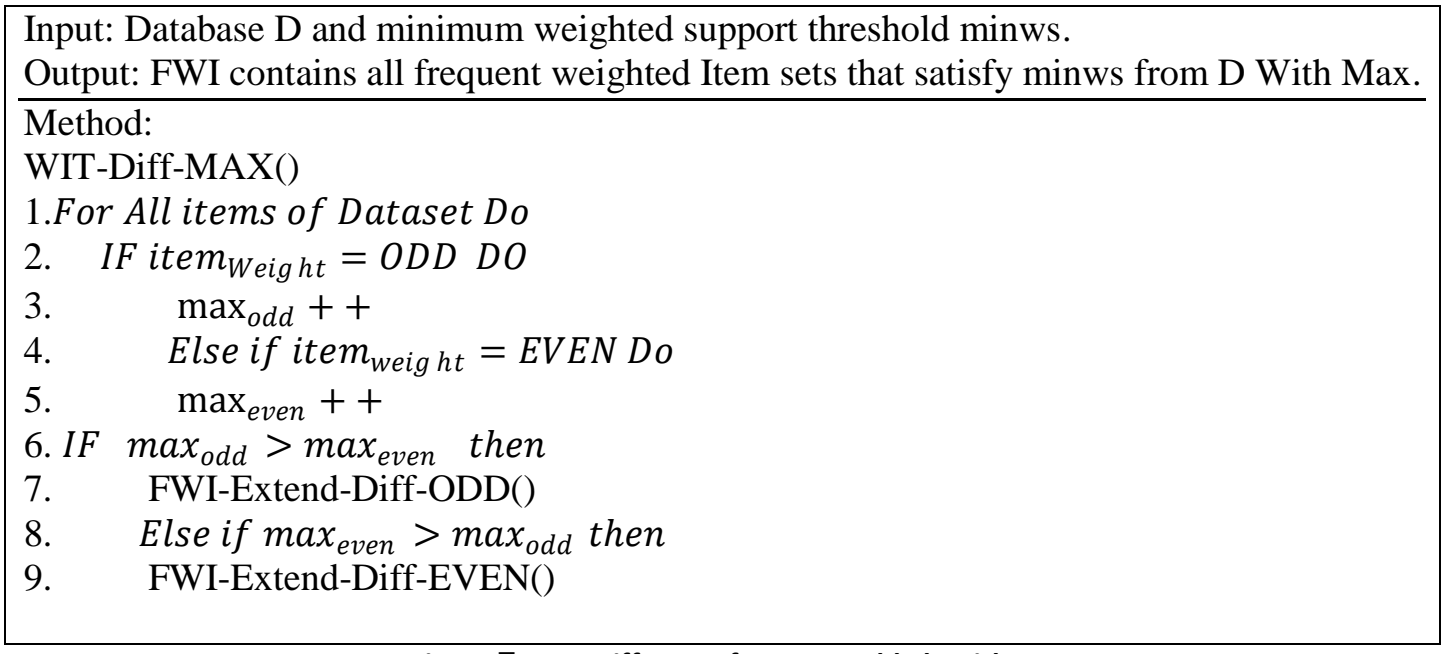

Figure 7: WIT-Diff-max of even or odd algorithm

\subsection{WIT-Percent method:}

In third method, In first counting each of items in total dataset and then compute percent of each item in all items. And then run method with specified percent of items. (Fig 8)

Input: Database D and minimum weighted support threshold minws.

Output: FWI contains all frequent weighted Item sets that satisfy minws from D.

Method:

WIT-Diff-Percent ( )

Array 1[ ][ ]= count of all items.

Array 2[ ][ ]=percent of each items in dataset.

Computing Percent of each item in dataset.

Input user threshold for percent of items.

1. $L_{r}=$ All items that their ws satisfy minws.

2. sort Nodes in $L_{r}$ increasing by their ws. 


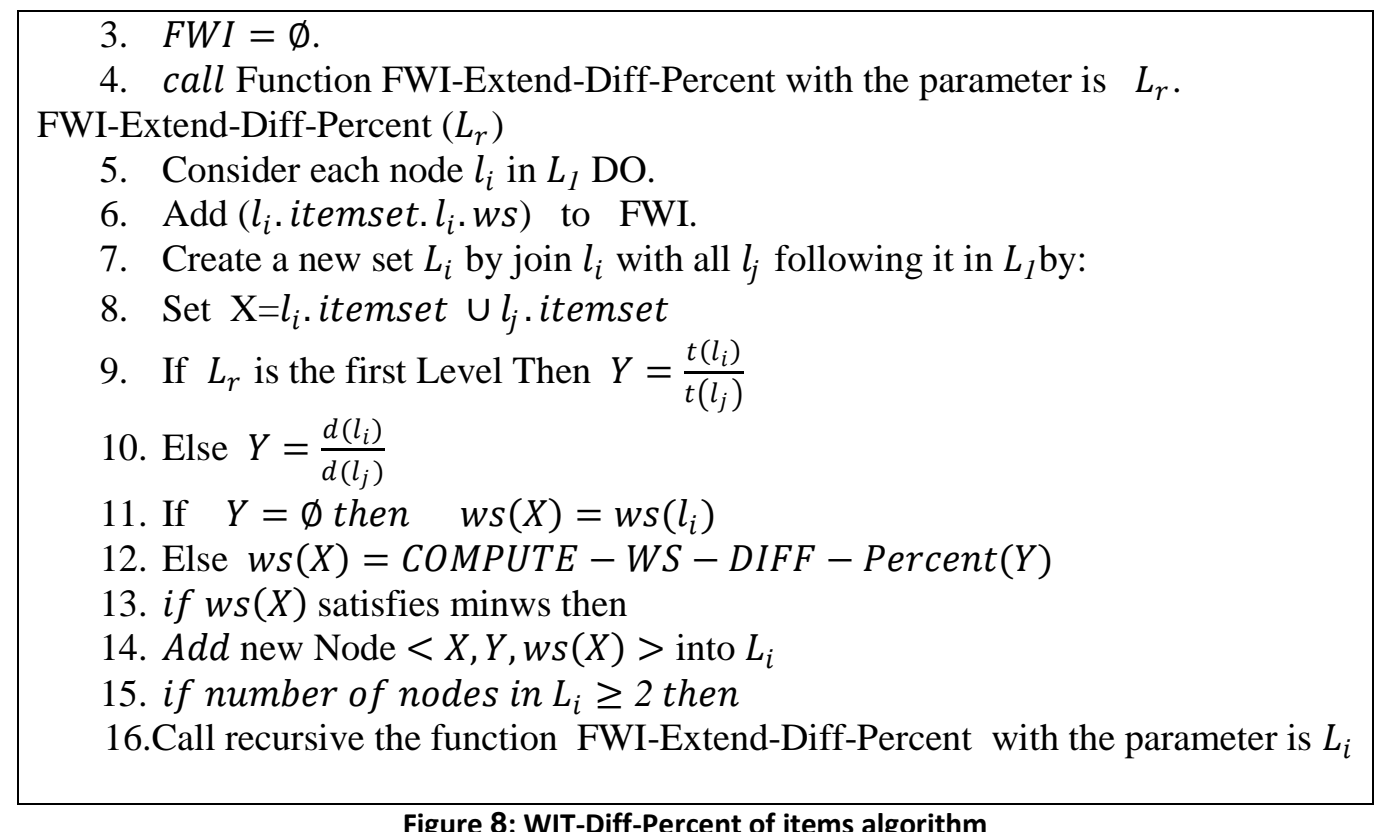

We can use data mining tools (such as Clementine software) for acquire count of items and percent of items in total of datasets. (See Fig 9 and Fig 10)

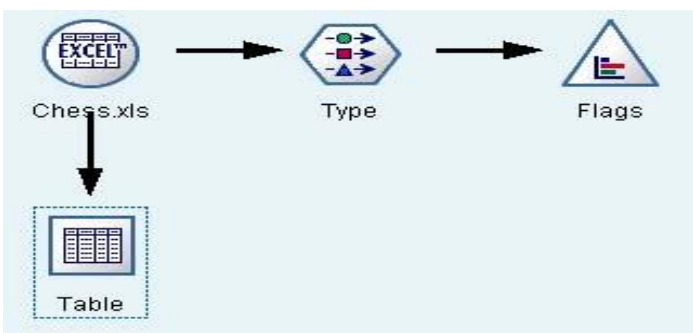

Figure 9: Clementine workspace

\begin{tabular}{|c|c|c|c|}
\hline Field & Proportion True & $\%$ & Count $r$ \\
\hline 1.000000 & & 47.79 & 1527 \\
\hline $13.000000=$ & 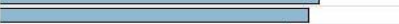 & 46.13 & \\
\hline $23.000000=$ & 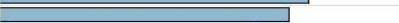 & 43.16 & 1379 \\
\hline $50.000000=$ & & 38.22 & \\
\hline $19.000000=$ & & 38.06 & 1216 \\
\hline $68.000000=$ & 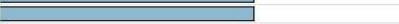 & 37.93 & 1212 \\
\hline $70.000000=$ & & 37.21 & 1189 \\
\hline $15.000000=$ & 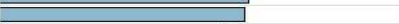 & 36.62 & 1170 \\
\hline $11.000000=$ & 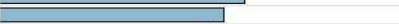 & 33.4 & 1067 \\
\hline $38.000000=$ & & 31.3 & 1000 \\
\hline 27.000000 & & 31.02 & \\
\hline $54.000000=$ & & 30.67 & \\
\hline $21.000000=$ & 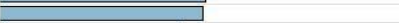 & 30.39 & \\
\hline 72.000000 & 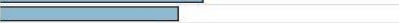 & 26.64 & \\
\hline 74.000000 & 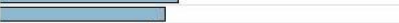 & 24.69 & 789 \\
\hline 17.000000 & 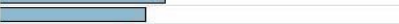 & 21.78 & 696 \\
\hline $46.000000=$ & 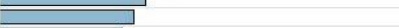 & 20.03 & 640 \\
\hline $44.000000=$ & 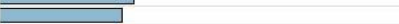 & 18.28 & 584 \\
\hline $64.000000=$ & 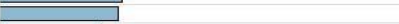 & 17.68 & 565 \\
\hline 42.000000 & 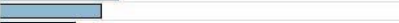 & 15.09 & 482 \\
\hline 3.000000 & 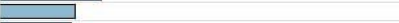 & 11.17 & 357 \\
\hline 25.000000 & 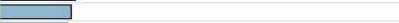 & 10.52 & 336 \\
\hline $9.000000=$ & & 10.08 & 322 \\
\hline $5.000000=$ & 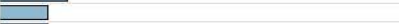 & 7.04 & 225 \\
\hline 31.000000 & & 7.01 & 224 \\
\hline $48.000000=$ & 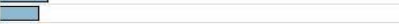 & 5.73 & 183 \\
\hline 56.000000 & & 5.48 & 175 \\
\hline $66.000000=$ & & 5.48 & 175 \\
\hline $34.000000=$ & & 4.88 & 156 \\
\hline 62.000000 & & 4.26 & 136 \\
\hline 7.000000 & & 3.76 & 120 \\
\hline $36.000000=$ & & 3.04 & \\
\hline ตก กถกกกกา & & 147 & \\
\hline
\end{tabular}

Figure 10: Out of Clementine workspace 


\subsection{WIT-Scope of weight method:}

In fourth method, use of domain for weight of items .as respects we assign for each item a value for weight with a random function .we can filter items with their weight and then running algorithm for selected items. In all method we purpose reducing the input of algorithm and as a result reduce run time and produce item sets. (Fig 11)

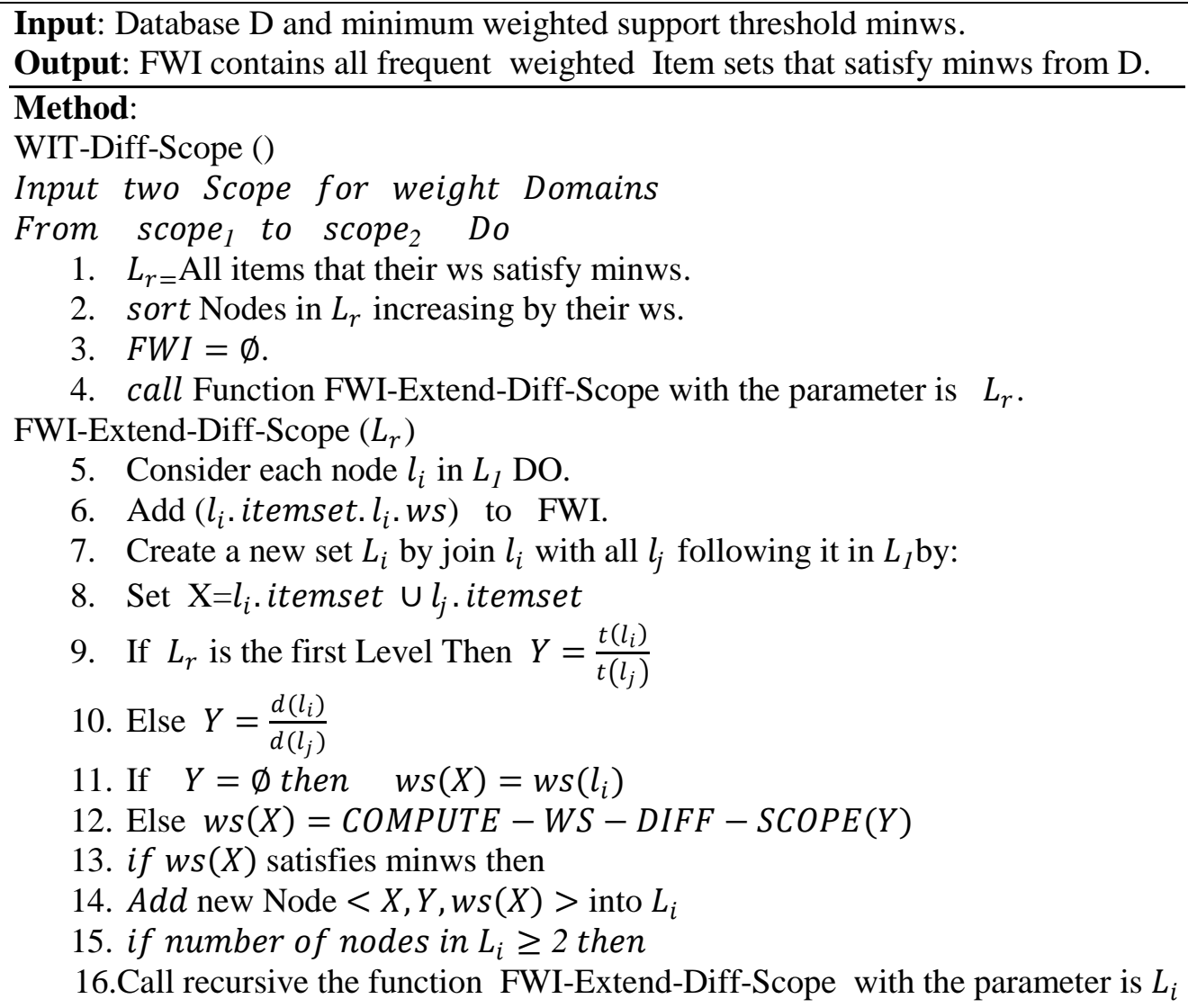

5. Consider each node $l_{i}$ in $L_{1}$ DO.

6. Add $\left(l_{i}\right.$. itemset. $\left.l_{i} . w s\right)$ to FWI.

7. Create a new set $L_{i}$ by join $l_{i}$ with all $l_{j}$ following it in $L_{l}$ by:

8. Set $\mathrm{X}=l_{i}$. itemset $\cup l_{j}$. itemset

9. If $L_{r}$ is the first Level Then $Y=\frac{t\left(l_{i}\right)}{t\left(l_{j}\right)}$

10. Else $Y=\frac{d\left(l_{i}\right)}{d\left(l_{j}\right)}$

11. If $Y=\emptyset$ then $w s(X)=w s\left(l_{i}\right)$

12. Else $w s(X)=C O M P U T E-W S-D I F F-S C O P E(Y)$

13. if $w s(X)$ satisfies minws then

14. Add new Node $<X, Y, w s(X)>$ into $L_{i}$

15. if number of nodes in $L_{i} \geq 2$ then

16.Call recursive the function FWI-Extend-Diff-Scope with the parameter is $L_{i}$

Figure 11: WIT-Diff-Domain of weight algorithm

\section{Experimental results}

All experimental described below were performed on a Intel(R) Core TM i5 $2.2 \mathrm{GHz}$.4GB RAM memory, Windows 7, using visual studio C\# 2010 the experimental datasets used for the experimentation were downloaded from http://finin.cs.helsinki.fi/data [14].we add a value for weight each of items with random function (values in the range of (1 to 10) for each datasets). In table 4 see more information of experimental datasets.

In table 4 view databases name and number of items and transactions, and in table 5 view result of run time of algorithms and methods. View number of FWI( Frequent weighted items) based MinWs threshold. 
Table 4: Information of datasets

\begin{tabular}{|l|c|c|l|}
\hline Database (DB) & \# Transactions & \#Items & Modified \\
\hline Chess & 3196 & 75 & Insert duplicate items on each transaction \\
\hline Mushroom & 8124 & 120 & Insert duplicate items on each transaction \\
\hline
\end{tabular}

Table 5: Number of FWI from databases

\begin{tabular}{|c|c|c|}
\hline Database & MinWs & \#FWI \\
\hline Chess & 80 & 8063 \\
\hline Chess & 70 & 16039 \\
\hline Chess & 60 & 23208 \\
\hline Chess & 50 & 29431 \\
\hline Mushroom & 50 & 436 \\
\hline Mushroom & 40 & 3038 \\
\hline Mushroom & 30 & 5347 \\
\hline Mushroom & 20 & 11634 \\
\hline
\end{tabular}

Table 6: Number of FWI with methods

\begin{tabular}{|c|c|c|c|c|c|c|}
\hline Database & MinWs & Even & Odd & Percent & Max & Domain of Weights \\
\hline Chess & 80 & 150 & 996 & $70 \% \rightarrow 5900$ & 996 & $(20: 90) \rightarrow 315$ \\
\hline Chess & 70 & 450 & 9376 & $60 \% \rightarrow 12912$ & 9376 & $(20: 90) \rightarrow 2955$ \\
\hline Chess & 60 & 3730 & 13815 & $50 \% \rightarrow 18126$ & 13815 & $(20: 90) \rightarrow 1043$ \\
\hline Chess & 50 & 5216 & 16057 & $40 \% \rightarrow 28422$ & 16057 & $(20: 90) \rightarrow 13817$ \\
\hline Mushroom & 50 & 18 & 17 & $80 \% \rightarrow 31$ & 18 & $(20: 90) \rightarrow 25$ \\
\hline Mushroom & 40 & 112 & 54 & $70 \% \rightarrow 31$ & 112 & $(20: 90) \rightarrow 42$ \\
\hline Mushroom & 30 & 133 & 71 & $40 \% \rightarrow 5331$ & 133 & $(20: 90) \rightarrow 91$ \\
\hline Mushroom & 20 & 689 & 134 & $30 \% \rightarrow 9452$ & 134 & $(20: 90) \rightarrow 336$ \\
\hline
\end{tabular}

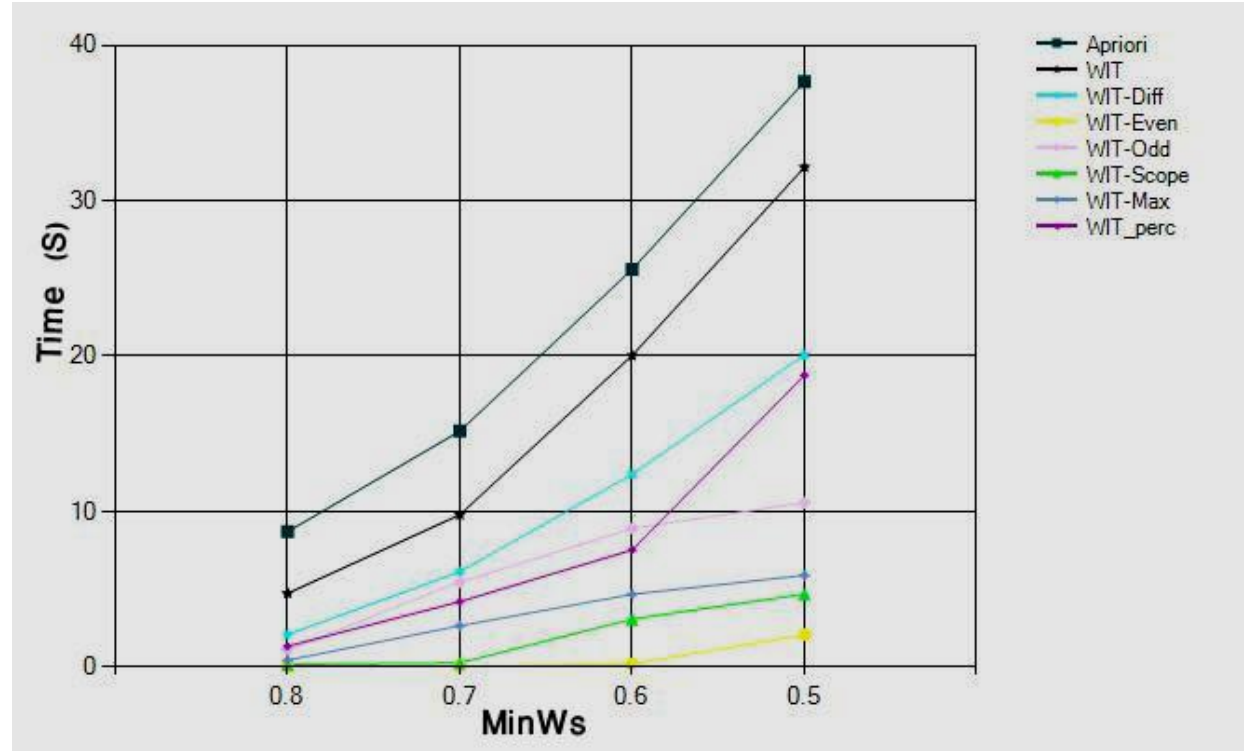

Figure 12: Run time for the eight methods in Chess dataset 


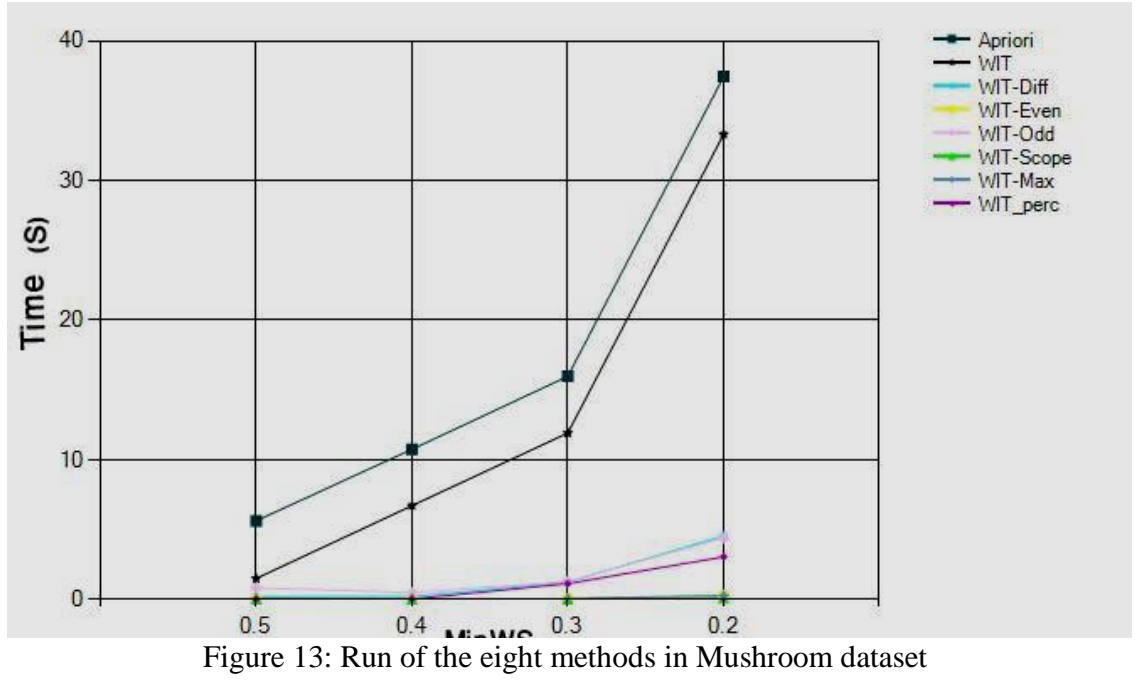

\section{Conclusion}

This paper has presented some method for mining frequent weighted item sets from weighted item transaction databases with reduce run time and reduce produce frequent item sets. And several efficient algorithms proposed. We use of WIT-Tree structure and apply my method to WIT-Diff algorithm that have less than run time from other algorithm. In this paper, we have concentrated only on the on the mining of FWIs using the proposed WIT-Tree data structure. And in my proposed methods at the first remove duplicated items in a transaction because, they not efficacy in computations and not compute similar transactions weight since used weight transaction of previous similar transaction.

\section{References}

[1]. A new method for mining Frequent Weighted. Vo, B,Frans Coenen \& Bac Le. 2013, VLDB94, pp. 489-499.

[2]. Fast algorithms for mining association rules. Agrawal, R., \& Srikant, R. 1994, VLDB'94, pp. 487499.

[3]. Mining association rules. Cai, C. H., Fu, A. W., Cheng, C. H., \& Kwong, W. W. 1998, international database engineering and, pp. 68-77.

[4]. An effective mining approach for up-todate. Hong, T. P., Wu, Y. Y., \& Wang, S. L. 2009, Expert Systems with Applications, pp. 9747-9752.

[5]. An efficient and effective association-rule. Hong, T. P., \& Wang, C. J. 2010, Expert Systems with, pp. 618-626. 
[6]. A weighted utility framework. Khan, M. S., Muyeba, M., \& Coenen, F. 2008, second uksim european symposium on, pp. 87-92.

[7]. A novel algorithm for mining high utility. Le, B., Nguyen, H., Cao, T. A., \& Vo, B. 2009, Asian conference on intelligent information and database, pp. 13-16.

[8]. An efficient strategy for mining high utility. Le, B., Nguyen, H., \& Vo, B. 2011, International Journal of Intelligent Information and Database Systems, pp. 164-176.

[9]. http://fimi.cs.helsinki.fi/data/. Dataset.

[10]. A dynamic bit-vector approach. Vo, B., Hong, T. P., \& Le, B. 2012, Expert Systems with Applications, pp. 7196-7206.

[11]. Interestingness measures for association rules. Vo, B., \& Le, B. 2011, Expert Systems with Applications, pp. 11630-11640.

[12]. Mining minimal non-redundant association rules. Vo, B., \& Le, B. 2011, Journal of Intelligent Systems Technology and Applications, pp. 92-106.

[13]. Mining association rules with weighted items. Cai, C. H., Fu, A. W., Cheng, C. H. 1998, international database engineering and, pp. 68-77.

[14]. A new methid for mining frequent weighted itemsets based on wit-trees. Vo, B,Frans Coenen \& Bac Le. 2013.

[15]. Mining non-redundant association rules. Zaki.M.J. 2004.

[16]. Weighted association rule mining using weighted support and significance framework.

Tao.F.Murtagh. 2003.

[17]. Weighted association rule mining via a graph based connectivity model. Russel Pears, Yun Sing

Koh,Gillian Dobbie,Wai Yeap. s.1. : Information Sciences, 2012.

[18]. Surveying Robot Routing Algorithms with Data Mining Approach. Rouhollah Maghsoudi, Somayye Hoseini. No.2, s.1. : The Journal of Mathematics and Computer Science, 2011, Vol. Vol .2.

[19]. Rule Extraction for Blood Donators with Fuzzy Sequential Pattern Mining. Fatemeh Zabihi, Mojtaba Ramezan, Mir Mohsen Pedram, Azizollah Memariani. 1, s.1. : The Journal of Mathematics and Computer Science, 2011, Vol. 2. 